\title{
A Novel Adaptive Routing and Switching Scheme for Software-Defined Vehicular Networks
}

\author{
Liang Zhao ${ }^{1}$, Weiliang Zhao ${ }^{1}$, Changqing Gong ${ }^{1}$, Ahmed Al-Dubai ${ }^{2}$, and Geyong Min ${ }^{3}$ \\ ${ }^{I}$ School of Computer Science, Shenyang Aerospace University, Shenyang, China \\ ${ }^{2}$ School of Computing, Edinburgh Napier University, UK \\ ${ }^{3}$ College of Engineering, Mathematics and Physical Sciences, University of Exeter, UK \\ Corresponding Author: Liang Zhao (1zhao@sau.edu.cn)
}

\begin{abstract}
Software-Defined Vehicular Networks (SDVNs) technology has been attracting significant attention as it can make Vehicular Ad Hoc Network (VANET) more efficient and intelligent. SDVN provides a flexible architecture which can decouple the network management from data transmission. Compared to centralized SDVN, hybrid SDVN is even more flexible and has less overhead. This hybrid technology can eliminate the burden on the central controller by moving regional routing tasks from the central controller to local controllers or vehicular nodes. In the literature, different routing protocols have been reported for SDVNs. However, these existing routing protocols lack flexibility and adaptive approaches to deal with changing and dynamic traffic conditions. Thus, this paper proposes a new software-defined routing method, namely, Novel Adaptive Routing and Switching Scheme (NARSS), deployed in the controller. This adaptive method can dynamically select routing schemes for a specific traffic scenario. To achieve this, this paper firstly presents a method for collecting road network information to describe traffic condition where the method extracts the feature data used to generate the routing scheme switching model. Secondly, we train the feature data through an artificial neural network with high training speed and accuracy. Finally, we use the model as a basis for establishing the NARSS and deploy it in the controller. Simulation results show that the proposed scheme outperforms the single traditional routing protocol in terms of both packet delivery ratio and end-to-end delay.
\end{abstract}

Keywords-VANET, hybrid SDVN, adaptive, routing scheme switching, artificial neural network

\section{INTRODUCTION}

Vehicular networking promotes the development of intelligent transportation system (ITS) with a wide range of applications, related to road safety, efficient traffic management and entertainments. In vehicular networks, each vehicle is equipped with an On-Board Unit (OBU) and support the communication architectures including vehicleto-vehicle (V2V), vehicle-to-infrastructure (V2I), vehicle-topedestrian (V2P), vehicle-to-network (V2N) which all together form the vehicle-to-everything (V2X). The applications of vehicular networks can be divided into safety applications and comfort applications. The safety applications provide the safe journey to passengers by sending and receiving information where such applications include lane change warning, collision information, emergency video streaming, and incident management. In contrast, the comfort applications are related to the comfort degree of traveler, to enable the services such as entertainment, electronic toll collection, parking lot payment. To meet the requirements and standards of these services, vehicular networks should provide efficient and high-quality communication, with reduced average end-to-end delay and improved packet delivery ratio.

As a current commercialized solution of vehicular network, dedicated short range communications (DSRC) has been widely applied in the US, Japan and Europe. However, DSRC only support one-hop while it is extremely dependent on the infrastructure. On the other hand, VANET has been proposed to expand the communication range of vehicles, an issue that widely studied over the last two decades. The major impediment of VANET arises from the vehicles dynamicity challenged by the difficulty to deal with the rapid changes of the network topology. Hence, researchers intend to find a new type of communication architecture or promote efficient communication schemes based on new network architectures. For instance, authors design a vehicular-based hierarchical software-defined architecture to improve performance in case of loss connection with the controller [1]. In [2], authors propose a $5 \mathrm{G}$ based SDN architecture for ITS which consists of three function layers, i.e., sensing layer, relay layer, and core network layer. In [3], 5G-SDN-VANET structures is used to implement social-aware clustering algorithm. Apart from these types, a UAV-VANET model designed by applying unmanned aerial vehicles as store-carry-forward nodes for vehicular networks [4].

In the light of the above, it is obvious that SDVN is a promising vehicular networking paradigm which can provide extensible and flexible means to manage networks to enable V2V and V2I communications. With SDVN, new routing schemes can be deployed easily. SDVN decouples the data plane and the control plane so that it can separate data forwarding functions and network functions. The SDVNbased architecture consists of two main components, controller and device. Devices transfer packets based on strategies dictated to each of them from the controller which has global knowledge about devices.

SDVN can be divided into two categories: centralized SDVN and hybrid SDVN. In centralized SDVN, central controller gathers the information of vehicles to construct a global view, then computes the optimal routing paths. Centralized SDVN relies on the processing of the central controller, which puts a heavy burden on the central controller and cause excessive network overhead. In contrast, hybrid SDVN spreads the burden of central controller to local controllers, which reduce the burden on central controller.

In VANET, different vehicular routing protocols demonstrate distinct performance abilities in different traffic scenarios. For example, AODV [5] performs well in urban scenes with high vehicle density, while GPSR [6] is more suitable for networks with sparse nodes and high speed of nodes. In order to select the most appropriate routing scheme in a specific geographical environment and traffic condition, we propose a new routing management method in hybrid SDVNs. We demonstrate how the method collect and process feature data, then establish a real-time decision-making model for routing. The key contributions are listed as follows:

- We propose a method for extracting road network information. By obtaining real-time road network 
information, road network characteristics and traffic conditions can be described.

- To the best of our knowledge, this work is the first to consider the adaptive routing in SDVNs to enable efficient communication for vehicles. A novel adaptive routing and switching scheme (NARSS) is proposed. We applied the OS-ELM for real-time model training. This kind of artificial neural network can train data chunk-by-chunk or one-by-one (a special case of chunk), so it can be used for real-time applications. It is a versatile solution that works for switching various routing schemes.

- We combine the hybrid SDVN architecture with NARSS. The local controller acts as a mobile edge node for collecting, processing the local feature data, and switching routing schemes based on the central controller's decision-making model. The central controller is responsible for merging the data and training the decision-making model in real time.

The rest of the paper is organized as follows. We summarize the related work in Section II. Section III introduces the adaptive routing and switching scheme, including the system model and the specific implementation method. In Section IV, the simulation process and the analysis of the result is discussed in detail. We conclude our work in Section V.

\section{RELATED WORK}

Recently, adaptive routing schemes and SDN-based routing schemes have been proposed for VANET. These studies have contributed to the development of vehicle communication and have implications for subsequent works. In general, routing protocols can be classified into two categories, namely topology-based routing protocols and position-based routing protocols [7]. AODV [5] and GPSR [6] are the representatives of these two types of routing protocols, respectively. In [8], authors combine the characteristics of reactive routing with position-based geographic routing in a manner that effectively uses all available position information. It can minimize the routing overhead and exit to reactive routing as position information degrades. In [9], ARPRL (adaptive routing protocol based on reinforcement learning) is proposed. Through distributed QLearning, ARPRL continuously learns and obtains the new link status of the network proactively with the periodic HELLO packets in the form of $Q$ table update. Hence, ARPRL has excellent adaptability to dynamic changes in the network. Authors present a bio-inspired unicast routing protocol called URAS [10]. URAS uses the cellular attractor selection mechanism to select next hops. Through routing feedback packets, it can adjust itself to adapt to dynamically changing environments. In [11], an adaptive quality-ofservice (QoS)-based routing for VANETs called AQRV is introduced. AQRV can adaptively select the intersections to reach the destination. The selected route satisfies the QoS constraints. In order to provide guaranteed quality-of-service (QoS) video-on-demand (VOD) sessions in VANET, a new adaptive geographic routing scheme is proposed in [12]. It discovers a number of independent routes between source and destination according to the volume of the requested video and lifetime. In order to ensure the quality of its services, it can estimate link stability by constructing link connectivity parameters.

It is clear that SDVN is an optimal architecture to allow the intelligent operation of adaptive routing strategy, in particular for vehicular networks. Huma Ghafoor presents a cognitive routing protocol based on hybrid SDVN architectures. The main controller (MC) and multiple local controllers (LC) build global topology view and local topology view respectively. Two nodes can only communicate when they have consensus about a common idle channel [13]. In [14], a SDVN-enabled connectivityaware geographical routing protocol (SCGRP) of VANET is introduced. The routing process is calculated by the remote controller. The controller only calculates the routing metrics for efficient routing decisions. SDN-based Vehicle Ad-hoc On-demand Routing Protocol (SVAO) [15] uses a two-level structure, including centralized local level consisting of vehicles and distributed global level consisting of local controllers. Since SVAO is designed building on AODV, and deploys the global view of SDVN to achieve centralized control, this scheme can improve system performance. The authors in [16] use the architecture of 5G-VANET-SDVN to achieve adaptive vehicle clustering and beamformed transmission for aggregated traffic. With the assistance of SDVN, the arriving road traffic can be predicted to adaptively implement vehicles clustering. The cluster head aggregates the traffic of each vehicle and communicates with the cellular BS, which can reduce the overhead. The paper also proposed a dual $\mathrm{CH}$ design to ensure seamless trunk link communication and robustness. In [17], the study comes up with an SDVN-based geographic routing protocol for VANET (SDGR). Based on the global view of controller, SDGR applies the electronic map, the location of the nodes and the collected vehicle density to calculate the optimal route. Also, by adopting the controller, the proposed method [18] can reduce congestion of $\mathrm{V} 2 \mathrm{~V}$ communications while routing data on road segments. In [18], the routing mechanism considers other existing routing paths which are relaying data in VANETs. The mechanisms deployed in the controller enable congestion control and detect wave connectivity. Based on these operations, it can optimize the resources configuration.

In general, adaptive or hybrid routing strategies improves the traditional routing protocols by applying intelligent algorithm or combining the advantages of several routing algorithms. However, a specific routing scheme can only be applied to a few traffic scenarios. Hence, the flexibility and adaptability of these routing strategies is limited. In contrast, the SDVN provides a new architecture for vehicle communication. SDVN controllers can provide a global view to optimize traditional routing. Even if the SDVN architectures are applied, these SDVN-based protocols have different performance in different traffic scenarios. Considering these circumstances, we propose a novel adaptive routing scheme switching method based on the SDVN architecture to select optimal routing scheme in a specific scenario.

\section{ADAPTIVE Routing AND SWITCHING ScHEME}

\section{A. System Model}

In SDVNs, each vehicle is equipped with a transceiver for short-range wireless communication, such as a DSRC device. Location and speed of each node can be obtained through the global positioning system (GPS). Apart from this, through electronic maps, we can acquire various feature data for describing geographic features and real-time traffic conditions. We use the hybrid SDVN architecture to implement real-time routing scheme switching. Hybrid SDVN spreads the burden of the central controller to the local controller. The local controller acts as a mobile edge 
computing (MEC) node. Based on hybrid SDVN, NARSS is composed of the OS-ELM [20], data collection and processing methods. It is a versatile solution to adaptively select the optimal routing strategy based on road network characteristics and real-time traffic conditions. The routing scheme switching method is deployed in the controller of SDVN.

In order to achieve regional management, we apply Geohash [19] to partition the area and deploy a local controller for each area. Geohash is a geographic coordinate system that divides map into grid-shaped blocks. It uses a string to represent the coordinates of longitude and latitude. Fig. 1 shows an example of Geohash partition, in which the region is near the Beijing Station, China. The node in a certain area converts the latitude and longitude information into a Geohash value, and this value can uniquely identify the area where the node is located. In this case, node can transfer the location and speed information to a specific local controller. Local controller constructs the simulation scenario through the collected data to derive the performance of different routing policies in this scenario. Then the optimal routing policy is selected as the label of the feature data entry.

The central controller aggregates and trains the labeled data of each local controller. When the training is completed, the central controller will send the decision-making model to the local controllers. As the local controller receives the model, it combines this decision-making model with the realtime local traffic conditions and the geographic features to select the optimal routing strategy. Then, the local controller broadcasts the messages containing flag and the decision of optimal routing strategy to all nodes.

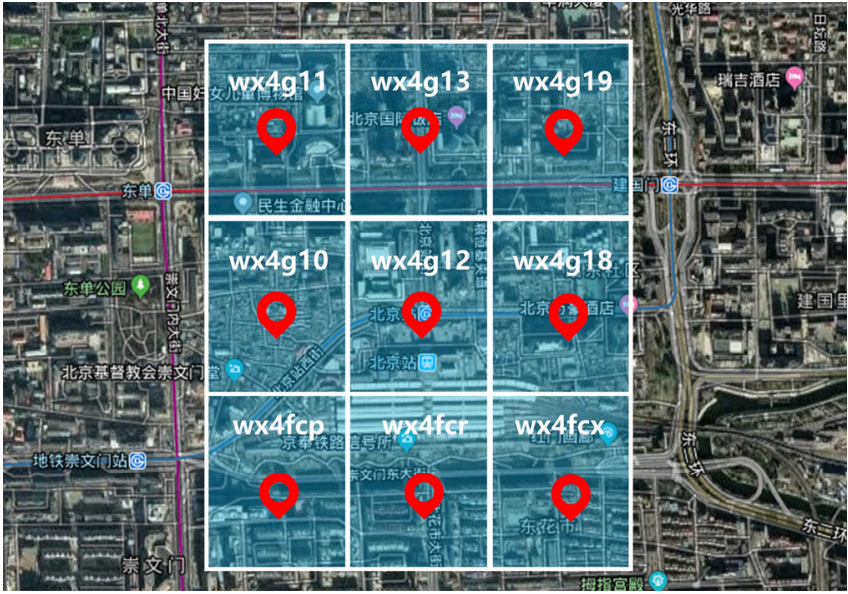

Fig. 1. Geohash area near the Beijing Station

Fig. 2 shows the system model which is based on hybrid SDVN. In this kind of hybrid SDVN, the local controller acts as a server node of MEC for processing real-time traffic information, switching the routing schemes through the decision-making model trained and provided by central controller, and sends labeled historical data to the central controller. Meanwhile, the central controller is deployed to train the decision-making models, and send new decisionmaking model to all local controllers. OS-ELM deployed in the central controller does not need to merge the historical data and the new data for unified training, but only needs to adjust the decision-making model through the new data to adapt to the real-time dynamic traffic conditions, thus it can greatly reduce the cost.

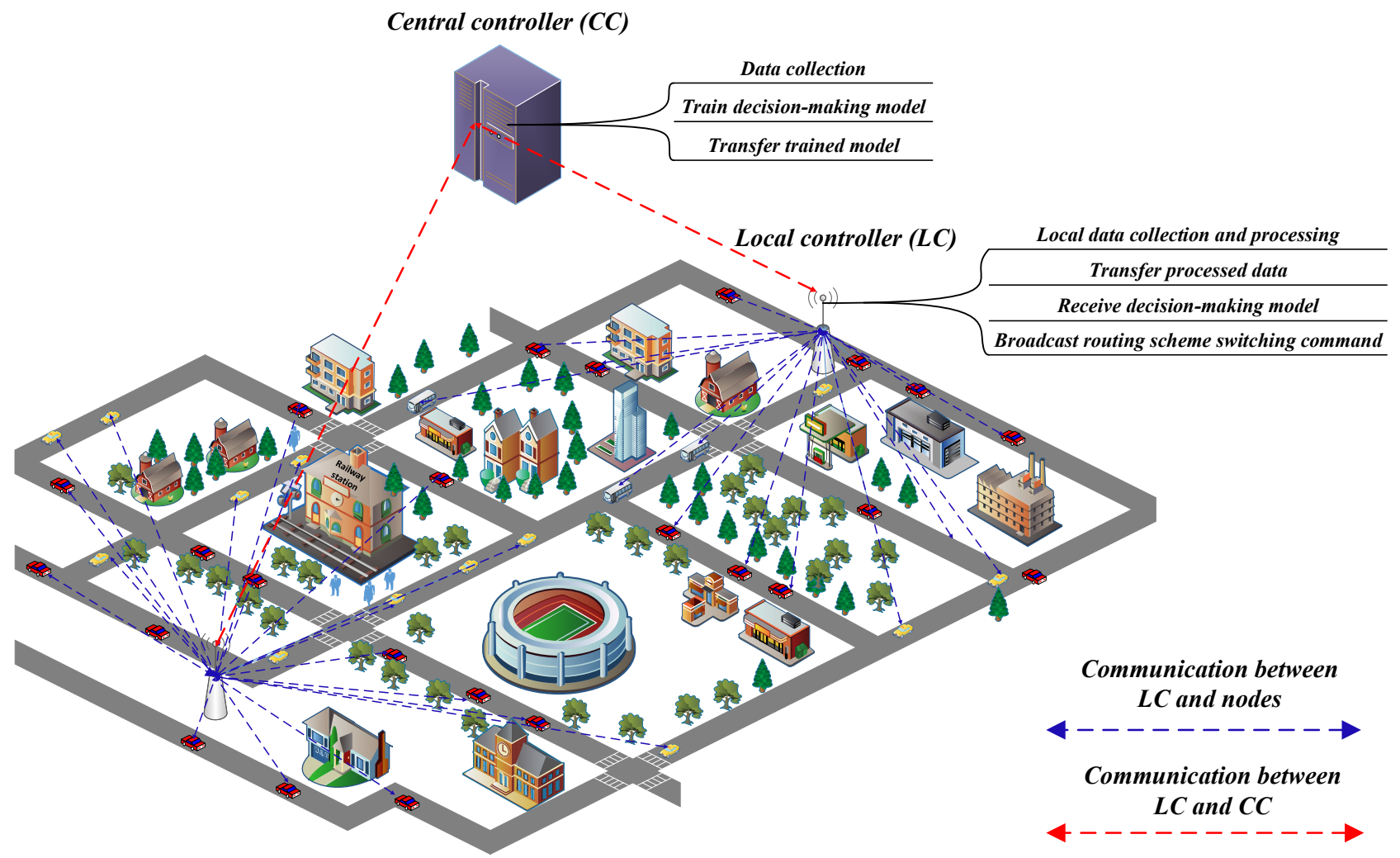

Fig. 2. Framework of NARSS system 


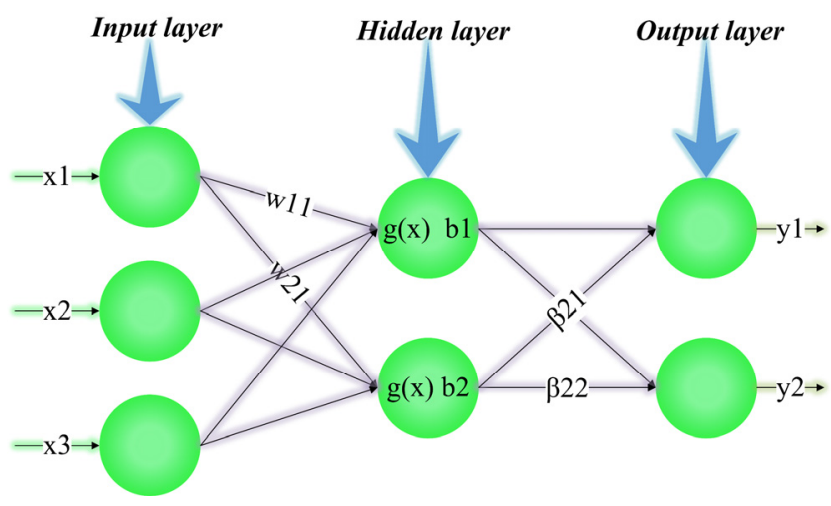

Fig. 3. Example structure of ELM

\section{B. ELM v.s.OS-ELM}

ELM is a Single-hidden Layer Feedforward Neural Network (SLFN) [21]. The structure of the ELM neural network is shown in Fig.3. This kind of artificial neural network is constructed by an input layer, a hidden layer and an output layer. Hidden layer contains the activation function and the operation of bias. The ELM algorithm randomly generates the connection weights of the input layer to the hidden layer and the biases of the hidden layer neurons. No manual adjustment parameters are required during training process. Only the number of hidden layer neurons needs to be set before training. In supervised batch learning, ELM applies a finite number of labeled data for training. According to the principle of ELM, we get equation (1)

$$
\mathrm{H} \beta=\mathrm{T}
$$

$\mathrm{H}$ is the hidden layer output matrix of the network. $\beta$ is the weight matrix between hidden layer and output layer. We can get $\beta$ by matrix operation. It can be estimated as

$$
\hat{\beta}=\mathrm{H}^{\dagger} \mathrm{T}
$$

We can use the derived output weights $\beta$ to act on the new data set to predict the labels of the data entries. Compared with previous traditional training methods, the ELM method has the advantages of fast learning speed and excellent overall performance. However, the data will be updated regularly in the actual application scenario. Data arrive chunk-by-chunk or one-by-one. Hence, ELM should be modified to make it online sequential.

On the contrary, OS-ELM [20] is an online sequential learning algorithm for single hidden layer feedforward networks. OS-ELM is based on ELM to achieve online learning. It can learn data chunk-by-chunk (a block of data) or one-by-one with a fixed or varying chunk size. When new data entries arrive, the training process does not incorporate historical data for retraining, but, rather, only trains new data to fine tune the output weights matrix. The learning algorithm has no prior knowledge about training observations times that will be presented. Compared with deep learning, ELM and OS-ELM present faster training speed while the prediction accuracy is better than deep learning in many application scenarios. OS-ELM realizes online learning. This makes it possible to implement problems in many online scenarios. We assume that the initial training data set and another chunk of data are defined as $\Upsilon_{0}$ and $\Upsilon_{1}$, respectively. $N_{i}$ is the number of data entries.

$$
\Upsilon_{0}=\left\{\left(x_{i}, t_{i}\right)\right\}_{i=1}^{N_{0}} \quad \Upsilon_{1}=\left\{\left(x_{i}, t_{i}\right)\right\}_{i=N_{0}}^{N_{0}+N_{1}}
$$

Then, the problem becomes minimizing (4), $H_{0}$ and $T_{0}$ are obtained by matrix operation of initial data while $H_{1}$ and $T_{1}$ are obtained by matrix operation of new data.

$$
\left\|\left[\begin{array}{c}
\mathrm{H}_{0} \\
\mathrm{H}_{1}
\end{array}\right] \beta-\left[\begin{array}{l}
\mathrm{T}_{0} \\
\mathrm{~T}_{1}
\end{array}\right]\right\|
$$

Through a series of derivations, we can get the recursive formula of sequential learning phase.

$$
\begin{gathered}
\mathrm{P}_{k+1}=\mathrm{P}_{k}-\mathrm{P}_{k} \mathrm{H}_{k+1}^{\mathrm{T}}\left(\mathrm{I}+\mathrm{H}_{k+1} \mathrm{P}_{k} \mathrm{H}_{k+1}^{\mathrm{T}}\right)^{-1} \mathrm{H}_{k+1} \mathrm{P}_{k} \\
\beta^{(k+1)}=\beta^{k}+\mathrm{P}_{k+1} \mathrm{H}_{k+1}^{\mathrm{T}}\left(\mathrm{T}_{k+1}-\mathrm{H}_{k+1} \beta^{k}\right)
\end{gathered}
$$

where $\mathrm{P}_{k+1}=\mathrm{K}_{k+1}^{-1}, \mathrm{~K}_{k+1}$ can be obtained from the recursion formula of $\mathrm{K}_{i}$. The recursion process of $\mathrm{K}_{i}$ is shown in Equation (7) and Equation (8). The specific derivation process can be found in the literature [20].

$$
\begin{gathered}
\mathrm{K}_{0}=\mathrm{H}_{0}^{\mathrm{T}} \mathrm{H}_{0} \text { and } \mathrm{K}_{1}=\mathrm{K}_{0}+\mathrm{H}_{1}^{\mathrm{T}} \mathrm{H}_{1} \\
\mathrm{~K}_{k+1}=\mathrm{K}_{k}+\mathrm{H}_{k+1}^{T} \mathrm{H}_{k+1}
\end{gathered}
$$

We can update the output weights matrix $\beta$ by Equation (6). It enables predictive analysis through real-time data.

\section{Collect Road Network Feature Data}

The formation of the existing road network is gradually evolved under the conditions of certain social and historical conditions. There are many forms of urban road network. In this paper, we consider four typical structures of road network, including grid road network, radial-circular road network, strip road network and hybrid road network.

Grid road network is the most common type of road network layout. The geometric figure is substantially a regular rectangle, that is, a nearly parallel backbone road is set at a certain distance, and subsidiary roads is arranged between the backbone roads. Radial-circular road network is gradually developed from the center of the city. It consists of several radiation lines from the center of the city and several circular lines centered on the city centre. Strip road network usually uses a backbone road as the axis, industrial and residential buildings are arranged along both sides of the line. Some subsidiary roads are connected from the backbone road to each side of the building group. Hybrid road network is a combination of various road network forms, which not only promotes the advantages of each road network form, but also avoids their shortcomings. With the development of modern urban economy, the scale of cities has been expanding, more and more cities have developed in this direction. Fig.4 shows the structure of the above four typical road networks. The upper left corner is radial-circular road network; the lower left corner is hybrid road network; the upper right corner is strip road network; and the lower right corner is grid road network.

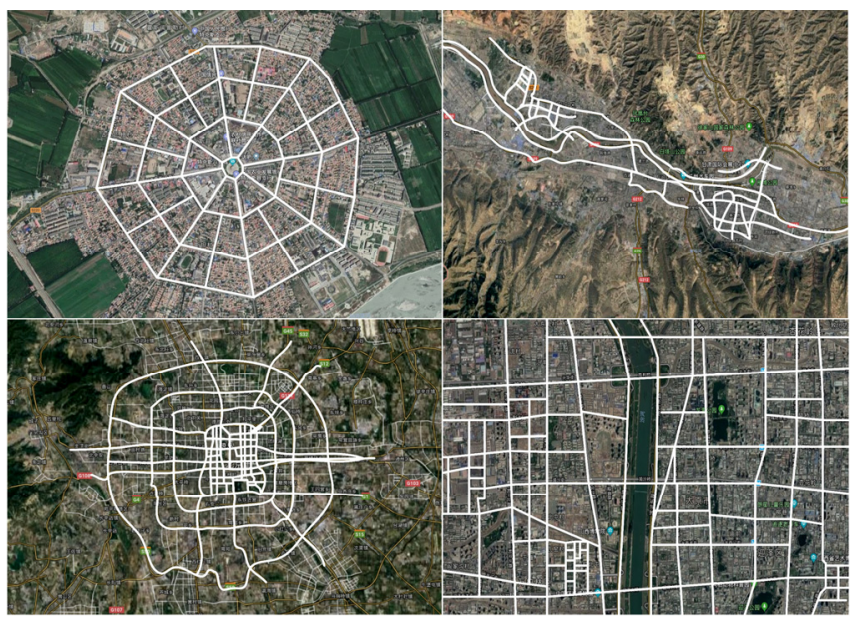

Fig. 4. Four typical road network structures 
In the actual traffic environment, there are various parameters could influence the performance of the routing protocol. Therefore, we extract the road network information and real-time traffic information to describe the traffic situation. Table I defines the various road network features. $R N_{\text {type }}$ is the road network category, including the above four types. $N_{i}$ is the number of intersections. When vehicles travels to the intersection, they will slow down, and traffic congestion may occur. $N_{t l}$ denotes the number of the traffic lights. Traffic lights are generally set at intersections. When the red light is on, the vehicles gather near the intersection. The density of vehicles at both ends is high. On the contrary, the density of vehicles in the middle section is low. This situation has different effects on various routing protocols. $N_{v}$ is the number of vehicles. $R_{t l}$ is the total length of the roads. We can express the average density of vehicles as shown in equation (9).

$$
D_{c}=N_{v} / R_{t l}
$$

Within a certain range, when the $D_{c}$ increases, the average distance between the vehicles is reduced, which is more conducive to the establishment of the communication link. $R_{m s}$ is the maximum speed of vehicles that on a specific road segment. Since the speed of nodes are generated according to the normal distribution with the limit of $R_{m s}$, $R_{m s}$ can reflect the speed characteristics of nodes. $N_{r l}$ is the number of lanes. One-way road and two-way road have different effects on routing. One-way roads can only send packets in the direction of nodes movement, while two-way roads can send packets in the opposite direction. $S_{m}$ is the area of the map. We can express the density of roads as equation (10)

$$
D_{r}=R_{t l} / S_{m}
$$

A greater value of $D_{r}$ can lead a reduction of average interval of road segments. When the distance between different road segments is within the communication range of the nodes, packets can be forwarded across road segments.

TABLE I. FEATURE DEFINITION

\begin{tabular}{c|c}
\hline Feature & Definition \\
\hline Road network category & $R N_{\text {type }}$ \\
Number of intersections & $N_{i}$ \\
Number of traffic lights & $N_{t l}$ \\
Number of vehicles & $N_{v}$ \\
Maximum speed of roads & $R_{m s}$ \\
Total length of roads & $R_{t l}$ \\
Number of road lanes & $N_{r l}$ \\
Area of the map & $S_{m}$ \\
Density of vehicles & $D_{c}$ \\
Density of roads & $D_{r}$ \\
\hline
\end{tabular}

\section{Simulation Results}

In this section, a simulation of NARSS is presented on the NS3 platform [22]. Despite the fact that our general routing switching scheme, NARSS can be applied to any routing, however, this study consider two well know routing protocols. In other words, in order to clearly demonstrate the performance of NARSS, we adopt two of the most classic routing protocols, namely topology-based routing protocol AODV and position-based routing protocol GPSR.

\section{A. Simulation and Parameter Setup}

In the simulation scenario, we use SUMO [23] to generate maps or process existing maps downloaded from OpenStreetMap, and then generate nodes' movement files. In order to collect the initial feature data sets for training the decision-making models, we simulated the above four types of maps, including the real maps, and the artificial maps constructed on the basis of the characteristics of a particular type of map. Within the limited range in Table II, we randomize the various parameters in the simulations to ensure the diversity of data. For a map with the same distribution of roads, the area can influence the distance of adjacent roads. If the distance between the roads is within the communication range of the vehicles, the node can transmit packets across the road segment. In a certain area, the number of vehicles determines the density of vehicles which can directly affect the quality of packet transmissions. Thus, we limit the maximum speed on the road and the speed of nodes on the road segment is generated according to the normal distribution. In addition, we also randomly set the number of traffic lights, and the number of lanes. For the same traffic scenario, we have done a large enough amount of simulation to obtain the average packet delivery ratio and average end-to-end delay through randomly specify communication nodes. The detailed simulation parameters are listed in Table II. After collecting and processing the data for the initial training, NS3 and SUMO is then applied for co-simulation.

TABLE II. PARAMETERS DEFINITION

\begin{tabular}{c|c}
\hline Description & Value \\
\hline Simulation area & $1 \sim 10 \mathrm{~km}^{2}$ \\
Number of vehicles & $50 \sim 350$ \\
Number of lanes & 1,2 \\
Max speed in road & $30 \sim 80 \mathrm{~km}^{2} / \mathrm{h}$ \\
Transmission range & $250 \mathrm{~m}$ \\
Packets generation speed & $1 \sim 5$ packets $/ \mathrm{s}$ \\
Packets size & $1024 \mathrm{bytes}$ \\
communication nodes rate & $20 \%$ \\
MAC protocol & $802.11 \mathrm{p}$ \\
Data rate & $6 \mathrm{Mps}$ \\
\hline
\end{tabular}

The local controller can be divided into two execution modules. In the first execution module, the local controller collects the traffic condition feature data in real time. Based on the decision-making model from central controller, the optimal routing strategy can be selected, and distributed to the local controller. Then, when the local controller receives the new strategy, it broadcasts this decision model to others and vehicles. In the second execution model, the local controller uses the movement files from the previous time period for simulation. This feature data item is labeled by applying the simulation results. Then, local controller transfers it to the central controller. The central controller tunes the initial decision-making model based on the labeled feature data. After the training process, the central controller transfers the new decision model to the local controllers. The entire process completes the real-time update of the decision-making model.

We observe that during the evening peak hours, high density of vehicles may lead to low vehicular moving speed, and even traffic congestion. In off-peak hours, low density of vehicles allows vehicles to travel at high-speed. Based on this reality, we set up experimental scenarios in various types of maps to examine the performance of NARSS.

\section{B. Performance Metrics}

Packet Delivery Ratio (PDR): PDR is the ratio of the received data packets (received by the destination nodes) to the total number of packets sent by the source nodes during the simulation time.

Average End-to-End delay (AEED): AEED refers to the average time between the source nodes send packets and the destination nodes receive corresponding packets. 


\section{Simulation Results and Performance Analysis}

Fig. 5 shows the PDR of simulations in various types of road networks under different traffic parameters. From Fig.5, we can see that the PDR of NARSS is similar to AODV under different road conditions. Since the overall performance of AODV is better than GPSR in most traffic scenarios [9], NARSS will choose AODV in most cases which leads to the average PDR of NARSS similar to AODV. Compared with AODV, GPSR demonstrates lower AEED in most traffic conditions. NARSS chooses the optimal routing strategy corresponding to different traffic conditions. Therefore, we can find out the AEED of NARSS is between AODV and GPSR in Fig.6.

From the performance parameters of PDR and AEED, we can observe NARSS can effectively switch routing strategies by using the real-time traffic information. AODV performs better than GPSR in most scenarios [9]. Even in the scenarios where GPSR performs better, GPSR does not show a huge advantage compared to AODV. Hence, the performance of NARSS cannot be fully utilized. NARSS will have better performance if we can apply several routing strategies have their obvious advantages in different traffic scenarios which is also a part of our future work.

\section{CONCLUSION}

This paper proposes a novel adaptive routing and switching scheme which is combined with SDVN. NARSS is a versatile method deployed in the central controller. We present a method for using feature data to describe road network and real-time traffic information. The local controller is used to collect, process feature data and transmit it to the central controller. Then, through the decision-making model trained by central controller and combined with real-time traffic data, system can select the most appropriate routing strategy. The simulation results show that the proposed SDVN-based routing scheme switching method can effectively select the optimal routing strategy.

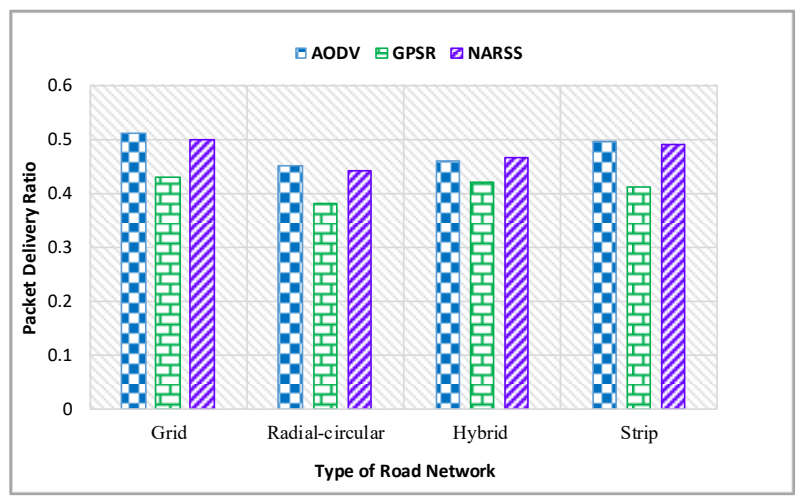

Fig. 5. Average packet delivery ratio versus road networks

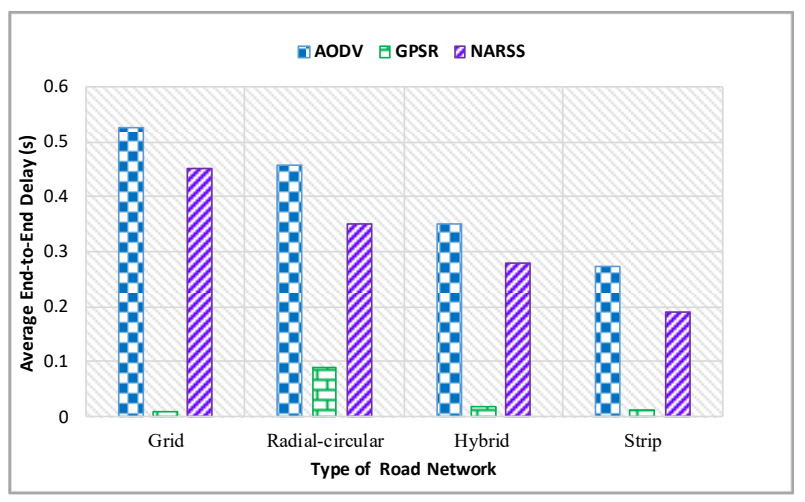

Fig. 6. Average end-to-end delay versus road networks

\section{REFERENCES}

[1] S. Correia, A. Boukerche and R. I. Meneguette, "An Architecture for Hierarchical Software-Defined Vehicular Networks," in IEEE Communications Magazine, vol. 55, no. 7, pp. 80-86, 2017.

[2] S. Din, A. Paul, A. Ahmad, S. H. Ahmed, G. Jeon and D. B. Rawat, "Hierarchical architecture for $5 \mathrm{G}$ based software-defined intelligent transportation system," IEEE INFOCOM 2018 - IEEE Conference on Computer Communications Workshops (INFOCOM WKSHPS), Honolulu, HI, 2018, pp. 462-467.

[3] W. Qi, Q. Song, X. Wang, L. Guo and Z. Ning, "SDN-Enabled SocialAware Clustering in 5G-VANET Systems," in IEEE Access, vol. 6, pp. 28213-28224, 2018.

[4] W. Fawaz, R. Atallah, C. Assi and M. Khabbaz, "Unmanned Aerial Vehicles as Store-Carry-Forward Nodes for Vehicular Networks," in IEEE Access, vol. 5, pp. 23710-23718, 2017.

[5] C. Perkins and E. Royer, "Ad hoc on-demand distance vector routing," in Proc. IEEE WMCSA, 1999, pp. 90-100.

[6] B. Karp and H. T. Kung, "GPSR: Greedy perimeter stateless routing for wireless networks," in Proc. 6th Annu. Int. Conf. Mobile Comput. New., Boston, MA, USA, Aug. 2000, pp. 243-254.

[7] B. Sharef, R. Alsaqour, M. Ismail, "Vehicular communication ad hoc routing protocols: A survey," in SienceDirect Journal of Network and Computer Applications, vol. 40, pp. 363-396, 2014.

[8] M. Al-Rabayah and R. Malaney, "A New Scalable Hybrid Routing Protocol for VANETs," in IEEE Transactions on Vehicular Technology, vol. 61, no. 6, pp. 2625-2635, July 2012.

[9] J. Wu, M. Fang, and X. Li. "Reinforcement Learning Based Mobility Adaptive Routing for Vehicular Ad-Hoc Networks." in Wireless Personal Communications, pp. 1-29, 2018.

[10] D. Tian et al., "A Microbial Inspired Routing Protocol for VANETs," in IEEE Internet of Things Journal, vol. 5, no. 4, pp. 2293-2303, Aug. 2018.

[11] G. Li, L. Boukhatem and J. Wu, "Adaptive Quality-of-Service-Based Routing for Vehicular Ad Hoc Networks With Ant Colony Optimization," in IEEE Transactions on Vehicular Technology, vol. 66, no. 4, pp. 3249-3264, April 2017.

[12] M. Asgharpoor Salkuyeh and B. Abolhassani, "An Adaptive Multipath Geographic Routing for Video Transmission in Urban VANETs," in IEEE Transactions on Intelligent Transportation Systems, vol. 17, no. 10, pp. 2822-2831, Oct. 2016

[13] H. Ghafoor and I. Koo, "CR-SDVN: A Cognitive Routing Protocol for Software-Defined Vehicular Networks," in IEEE Sensors Journal, vol. 18, no. 4, pp. 1761-1772, 15 Feb.15, 2018.

[14] D. K. N. Venkatramana, S. B. Srikantaiah and J. Moodabidri, "SCGRP: SDN-enabled connectivity-aware geographical routing protocol of VANETs for urban environment," in IET Networks, vol. 6, no. 5, pp. 102-111, 92017.

[15] S B. Dong, W. Wu, Z. Yang and J. Li, "Software Defined Networking Based On-Demand Routing Protocol in Vehicle Ad Hoc Networks," 2016 12th International Conference on Mobile Ad-Hoc and Sensor Networks (MSN), Hefei, 2016, pp. 207-213.

[16] X. Duan, Y. Liu and X. Wang, "SDN Enabled 5G-VANET: Adaptive Vehicle Clustering and Beamformed Transmission for Aggregated Traffic," in IEEE Comm. Magazine, vol. 55, no. 7, pp. 120-127, 2017.

[17] X. Ji, H. Yu, G. Fan and W. Fu, "SDGR: An SDN-Based Geographic Routing Protocol for VANET," 2016 IEEE International Conference on Internet of Things and IEEE Green Computing and Communications and IEEE Cyber, Physical and Social Computing and IEEE Smart Data, Chengdu, 2016, pp. 276-281.

[18] M. S. Rayeni and A. Hafid, "Routing in heterogeneous vehicular networks using an adapted software defined networking approach," 2018 Fifth International Conference on Software Defined Systems (SDS), Barcelona, 2018, pp. 25-31.

[19] Geohash Homepage, http://geohash.org/, [2018-9-7].

[20] N. Liang, G. Huang, P. Saratchandran and N. Sundararajan, "A Fast and Accurate Online Sequential Learning Algorithm for Feedforward Networks," in IEEE Transactions on Neural Networks, vol. 17, no. 6, pp. 1411-1423, Nov. 2006.

[21] G.-B. Huang, Q.-Y. Zhu, and C.-K. Siew, "Extreme learning machine:A new learning scheme of feedforward neural networks," in Proc.Int. Joint Conf. Neural Netw. (IJCNN2004), Budapest, Hungary, July 25-29, 2004, vol. 2, pp. 985-990.

[22] NS3 Homepage, https://www.nsnam.org/, [2018-9-7]

[23] SUMO Homepage, http://sumo.sourceforge.net/, [2018-9-7] 